Ueber das Verhalten des Phenols zu einigen Chinaalkaloïden :

\title{
von Demselber.
}

(Eingeluufen den 3. Juni 1876.)

In einer früheren Mittheilung *) wurde angegeben, dafs aus einer Auflösung von gleichen Mol.-Gew. Cinchonitin und Phenol in verdünntem Alkohol cine ölige Abschetidung erhalten werde, die sich allmälig in krystalle unselas und eine Phenolverbindung sei. Bei Anwendung von starkem Alkohol wird nun diese Verbindung sogleich in hübschen Krystallen erhalten, welche nach dem Abspülen mit verdünntem Alkohol vollkommen rein sind.

Diese Verbindung stellt farblose, glasglänzende, geruchlose Prismen dar, ist luftbeständig, entwickelt schon bei gelinder Wärnue Phenol und verliert dasselbe ganz, wenn sio auf etwa 130 " erhitzt wird.

0,7915 Grm. luftrockener Substanz gaben boi letzterer Temperatur 0,108 Phenol $=13,61 \mathrm{pC}$. Da die Vorbindung koin Krystallwasser enththt, so ergiebt sich aus dieser Bestimmung die Formel $2 \mathrm{C}_{90} \mathrm{H}_{4}, \mathrm{~N}_{2} \mathrm{O}, \mathrm{C}_{6} \mathrm{H}_{6} \mathrm{O}$, welche $13,25 \mathrm{pC}$. Phenol verlangt.

Diese Verbindung ist also Semiphenolcinchonidin und murs wohl als ein. Alkaloïd angesprochen werden, weil sich diese Substanz mit. Schwefelsäure zu dem früber schon beschriebenen Sulfat verbindet. Allein dieses Alkaloind ist im freien Zustande sehr unbeständig; denn ein wiederholtes Umkrystallisiren desselben aus Alkohol genügt schon, um alles Phenol daraus abzuscheiden.

Das Semiphenolcinchonidin reagirt in alkoholischer Lösung stark alkalisch. Wenn zu dieser Lösung sehr wenig Bisen-

*) Diese Anaalen I81, 56. 
chlorid gebracht wird, so fallt Eisenoxydhydrat nieder; bei weiterem Zusatz von Eisenchlorid wird aber, vielleicht unter Mitwirkung des abgeschiedenen Eisenoxydhydrats, eine dunkelgelbe Färbung der Lösung erzielt. Da wie erwähnt Alkohol aus der Verbindung eine gewisse Menge Phenol abscheidet, so folgt andererseits hieraus, dafs das Phenol unter den gegebenen Verhältnissen nicht aaf Eisenchlorid reagirt *).

Verdünnte Säuren, in Ueberschufs angewendet, zersetzen das Semiphenolcinchonidin, wobei Phenol abgeschieden wird, welches durch Ausschütteln der Lösung mil Aether gewonnen

*) Bekanntlich giebt die wisserige Phenollösung mit Eisenchlorid eine blauvioletto Furbun ${ }_{b}$, welche $H$. Schiff (diese Annalen 158 , 164) ala Beweis dafïr ansieht, dafs ein freits Phenolhydroxyl zagegen sei. Wenn überhaupt diese Hypothese begrïndet ist, so müfsten nach meiner Ansicht dio Grenzen, innerhalb welcher sie zutreffen soll, näher bestimnt werden, denn sie fallit unter Umathinden schon beim Phenol selbst. Roines Phenol giebt nümlich in alkoholiacher Lüsung nit Eisenchlorid keine Farbung; die gelbe Farbe, welche eine solche Lösung auf Zusatz von Eisenchlorid annimmt, ist nur vom Alkohol bedingt. Alkohol wirkt sogar der blanen Phenolreaction entgegen und hebt sio auf. Aus diesem Grunde verschwindet dic Farho der wässerigen, mit Eisenchlorid vermischten Phenollösung auf Zusatz von Alkohol. Auch das Morphinchlorhydral verhialt sich in dieser Woise ganz gleich wio Phenol. Dieses verschichene Verhalten von ein und derselben Substanz bei Anweudung verschiedener Lösungsmittel sollte meines Erachtons in deren Charakteristik mebr bervorgehoben werden, da bei Unkenntwifs dicser Verbültaisse leicht 'Lrugschlüsse gemacht werden kümucn. Ein Beispiel derart scheint neulich Ed. Hoff $m$ ann (Berichte der deutsclien chemischen Gesellschaft 8, 693) geliefert su haben. Derselbs giebt nämolich an, dafs sich das Aurantiin mit Eisenchlorid braumroth färbe, wabrend das dem Aurantiin offenbar kufserst uhnlicho Murrayin nach des Angaben von Blas mit Eisenchforid eine blaugrïne Farbenreaction zeige. Hoffman $n$ scheiut hieruach die Sulistanzen als bestimmt verschieden von einander $z$ balter. Bei alledem ist aber die ldentitat beider Substanzen woch fiir lien lall denkbar, dais jene Differenz in der Farbenreaction durch das angewardte Lösungamittel bodingt ist. 
werden kann. Nimmt man verdünnte Salpetersäure, so färbt sich die Lösung beim Erwärmen braunroth and giebt dann mil Ammoniak im Ueberschurs vermischt einen gelben Niederschlag.

Concentrirte Salpetersäure lōst das Semiphenolcinchonidin für den ersten Augenblick farblos, dann färbt sich diese Lösung gelb und schliefslich braunroth.

Eine andere Verbindung als die vcrgenannte, obwohl ihr im hohen Grade ahnlich, wird erhalten, wenn auf 1 Mol.-Gew. Alkaloin 2 hezw. 3 Mol.-Gew. Phenol genominen werden. Diese neue Verbindung, welcht in beiden Fällen resultirt, ist das Sesquiphenolcinchonidin.

Diese Substanz bildet farblose, glasglänzende, luftbeständige Krystalle, welche aber im Exsiccator während längerer Zeit etwas Phenol verlieren. Beim schwachen Erwärmen entwickeln dieselben intensiven Phenolgeruch und werden bei $130^{\circ}$ ganz frei von Phenol.

Von luftrockener Substanz gaben :

I. $0,4295 \mathrm{Grm}$. bei $130^{\circ} 0,133$ Phenol.

II. $0,3630, n, 0,111$, 0,5515 , 0,3797 Cinohonidin.

Dio Formel $2 \mathrm{C}_{80} \mathrm{H}_{2} \mathrm{~N}_{8} \mathrm{O}, 3 \mathrm{C}_{6} \mathrm{H}_{6} \mathrm{O}$

\begin{tabular}{lccc} 
& \multicolumn{2}{c}{ vetonden } \\
\cline { 3 - 4 } Phenol & verlangt & I & II. \\
Cinchonidin & 31,29 & 30,90 & 80,55 \\
& 68,71 & - & $68,72$.
\end{tabular}

Diest Verbindung scheint die höchste Sâttigungsstufe zwischen Cinchonidin und Phenol zu resprüsentiren; denn wenn diese Substanz zusammen mit viel Phenol in heifsem Alkohol gelöst wird, so krystallisirt gleichwohl nur Sesquiphenolcinchonidin. Von den erbaltenen Krystallen gaben :

0,4145 Grm. (lufttrocken) bei $130^{\circ}$ C. 0,1275 Phewal $=30,76 \mathrm{pC}$. (ber. 81,29 PC.). 
Das Sesquiphenolcinchonidin wird beirn Auflōsen in heifsem Alkohol ebenfalls zersetzt. Wird das Gewichtsverhältnifs zwischen Substanz und Alkohol etwa wie $1: 5$ eingehalten, so krystallisirt beim Erkalten der Lösung fast reines Semiphenolcinchonidin (gef. 16,62 pC. Phenol). Beim weiteren Unkrystallisiren der Substanz wird deren Phenolgehalt inehr und mehr herabgedrückt und schliefslich reines Cinchonidin erhalten.

Wird die heifse alkoholische Lösung der Sesquiverbindung genau mit schwefelsaure gesâltigt, so krystallisirt beim Erkalten derselben die Verbindung $2 \mathrm{C}_{40} \mathrm{H}_{x 4} \mathrm{~N}_{2} \mathrm{O}, \mathrm{C}_{8} \mathrm{H}_{6} \mathrm{O} . \mathrm{SO}_{8}$ $+5 \mathrm{H}_{2} \mathrm{O}$.

Während sich das Cinchonidin sehr leicht mit Phenol verbindet und diese Eigenschaft mit dem Chinin gemein zu haben scheint, sind andererseits dip nach rechts drehenden krystallisirbaren Chinaalkaloĭde : das Conchinin, Cinchonin und Chinamin nicht fähig, im freien Zustande eine Verbindung mit Phenol einzugehen. Wie auch das Verhältnifs zwischen Phenol und dem Alkaloìd gewählt wurde, immer schied sich dus letztere aus der alkoholischen Lösung frei von Phenol ab.

\section{Bernerkungen über Cynanchol; von Demselben.}

Butler off ") bezeichnete unlängst mit dem Namen Cynanchol eine in Nadeln und Blättchen krystallisirende Sub-

*) Diese Annalen $180,352$. 